\title{
论 文
}

\section{基于重要性测度的一箭多星分离安全性分析}

\author{
田龙飞 ${ }^{1,2}$, 李华 ${ }^{1^{*}}$, 刘武 ${ }^{1,2}$, 刘勇 ${ }^{1}$, 胡海鹰 ${ }^{1}$, 李平付 ${ }^{1}$ \\ 1. 中国科学院微小卫星创新研究院, 上海 201203; \\ 2. 上海微小卫星工程中心, 上海 201203 \\ * E-mail: 1h_secm@163.com \\ 收稿日期: 2018-05-16; 接受日期: 2018-08-29; 网络版发表日期: 2019-03-07 \\ 国家重点研发计划(编号: 2016YFB0501100)资助
}

\begin{abstract}
摘要 对于一箭多星发射任务, 各颗卫星分离速度及分离角度可能存在一定误差, 使得分离后星间距离存在不确 定性, 从而影响分离安全性. 为提高一箭多星分离安全性, 利用重要性测度分析方法对卫星入轨分离后的星间距 离开展研究. 提出了基于移动最小二乘的重要性测度指标求解新方法, 数值算例表明新计算方法相比传统的 Monte Carlo方法和Saltelli方法效率更高. 针对分离后星间距离过于接近的情况, 重要性测度分析方法能够有效识 别出对星间距离影响较大的变量, 对这些变量的分布参数进行优化调整后, 星间最小距离明显增大且不确定性降 低, 从而保证了一箭多星的分离安全性.
\end{abstract}

关键词一箭多星, 分离安全性, 重要性测度, 移动最小二乘, 不确定性分析

\section{1 引言}

随着航天技术的进步和微小卫星技术的快速发 展，一箭多星已经成为航天发射活动普遍采用的方式. 星箭分离作为发射程序的最后环节，直接关系到发射 任务的成败. 近年来, 学者对分离安全性开展了广泛 的分析，谢长雄和徐月 同 $^{[1]}$ 对星间分离机构进行分析 设计, 并完成了试验校验; 滕来和金仲和 ${ }^{[2]}$ 对皮卫星分 离参数进行了优化, 提高了卫星分离精度. 针对卫星释 放分离后的距离安全性分析，研究人员则提出了一些 解析和半解析的方法 ${ }^{[3-5]}$. 在一箭多星发射任务中, 卫 星分离仿真分析较为复杂，必须确保分离后各颗卫星 之间的距离足够, 以保证安全性 ${ }^{[6]}$. 分离后卫星的运动
状态受到多方面因素影响，包括弹簧弹力、爆炸螺栓 冲击力、航天器质量特性、初始位置速度参数等. 传 统的仿真分析过程中通常将分离后卫星的速度方向与 角度取标称值，并在此基础上进行星间距离的仿真分 析，然而实际上每颗卫星分离速度与分离角度均会存 在一定误差，这将会导致分离后卫星与卫星之间的距 离存在不确定性. 某些时候这些误差会使得分离后一 段时间内卫星与卫星之间距离过小，威胁到航天器的 安全性. 因此, 有必要对不同的输入变量(包括每颗卫 星分离后的姿态角、相对速度等)的不确定性对最终 卫星之间距离不确定性的贡献大小进行分析研究，有 助于在设计过程中, 关注重要变量, 增大星间距离, 确 保分离安全性.

引用格式: 田龙飞, 李华, 刘武, 等. 基于重要性测度的一箭多星分离安全性分析. 中国科学: 技术科学, 2019, 49: 803-814 Tian L F, Li H, Liu W, et al. Study on safety of multi-satellite launch mission based on importance measure (in Chinese). Sci Sin Tech, 2019, 49: 803814, doi: 10.1360/N092018-00145 
在可靠性及安全性工程中, 研究输入变量不确定 性对输出响应量不确定性的影响是一项十分重要的内 容, 同时也是目前学术界研究的热点. 在这一方面, 灵 敏度分析作为一种十分有效的分析工具，在工程实际 问题中得到了广泛应用. 目前对灵敏度分析最为常见 的定义是由Saltelli等人 ${ }^{[7]}$ 在2002年提出的: “灵敏度分 析是研究不同的输入变量的不确定性对模型输出响应 量不确定性的贡献程度大小的学问”. McRae等人 ${ }^{[8]}$ 认 为灵敏度分析应该被分为两大类，即局部灵敏度分析 和全局灵敏度分析(亦称重要性测度分析), 其中局部 灵敏度分析只能够研究模型在某个输入变量变化, 而 其他输入变量确定时模型输出响应量的局部变化情 况. 而全局灵敏度分析能够从整体上考虑模型输入变 量的不确定性对模型输出响应不确定性的影响, 并对 模型的输入变量进行重要性排序 ${ }^{[9]}$. 显然, 全局灵敏度 分析所得结果更符合工程实际需要，因而在工程实际 问题中能够有更广泛的应用. Saltelli ${ }^{[10]}$ 认为理想的重 要性测度指标应满足 3 个条件: 全局性、可量化及与 模型无关，并基于此提出了相应的重要性测度指标; 近年来, 如Sobol ${ }^{,[11]}$, Helton等人 ${ }^{[12]}$, Borgonovo ${ }^{[13]}$ 提出 了各种重要性测度指标及相应的计算方法. 在众多的 重要性测度指标体系中，应用最为广泛的是基于方差 的指标, 因为方差是最为常见的一种描述变量不确定 性的指标, 这类重要性测度指标能够反映每个输入变 量在其全部取值变化范围内对输出响应不确定性的综 合影响 ${ }^{[7]}$. 基于方差的重要性测度指标的计算方法很 多, 且各有优缺点. 其中Monte Carlo数字模拟法最为 直观, 且易于编程实现, 但其需要采用双重抽样, 效率 十分低下. Saltelli ${ }^{[14]}$ 提出一种基于单重抽样的数字模 拟法, 使得重要性测度指标的求解效率得到提高, 但 对于复杂运算模型(例如卫星轨道外推), 这种指标计 算方法的计算代价仍过于高昂, 需要进一步发展高效 计算方法. 对于复杂运算模型的输入变量重要性测度 分析, 唯一可行的计算方式是基于代理模型(metamodelling)的方法，代理模型在重要性测度分析中的应用 可行性在文献[15]中得到了严格的证明，此种计算方 式采用替代模型对不同输入变量取值条件下的模型输 出响应进行估算, 极大减小了计算量 ${ }^{[16]}$. 本文采用移 动最小二乘拟合作为代理模型来计算基于方差的重要 性测度指标, 相比传统的最小二乘拟合方法, 移动最小 二乘拟合将样本空间内的样本点按其与当前拟合点距
离远近赋予不同的权重值, 与当前拟合点距离越近, 权 重越高. 移动最小二乘法对非线性模型具有很强的适 应性, 在样本量足够的情况下, 适合任意维数的模型 拟合且具有较高的精度 ${ }^{[17]}$.

本文采用基于方差的重要性测度指标对一箭多星 分离安全性进行分析, 对于卫星分离速度与分离角度 的设计标称值如何选取, 本文不作分析. 仅在设计标 称值选定的情况下，考虑分离速度和分离角度的不确 定性, 即将其设为随机变量, 对分离后星间距离进行 分析, 针对星间距离较小的情况, 开展基本变量重要 性测度分析, 识别出对星间距离不确定性贡献较大的 变量, 从而能够指导工程实际, 在设计生产过程中优 化重要变量, 尽量增加分离后的星间距离, 同时减小 不确定性.

\section{2 一箭多星分离安全性问题描述}

一箭多星发射任务需要保证入轨分离后各颗卫星 之间的相对距离, 而各卫星的分离角度和分离速度决 定了分离后卫星的运动轨迹及各颗星之间的距离. 实 际工程中, 受多方面因素影响, 分离角度及分离速度 存在一定的误差，导致分离后星间距离为一不确定量, 可能影响分离安全性.

本文以一箭四星分离为例进行分析, 四星轨道均 为 $700 \mathrm{~km}$ 太阳同步轨道. 按照常规的火箭飞行程序设 计, 假设四星的星箭分离时序如表1所示.

四星分离速度和分离角度的设计标称值如表2所 示, 将它们全部设置为随机变量, 并假设各变量相互独 立. 结合实际统计结果, 假设分离速度和分离角度均服 从正态分布, 其中分离速度的变异系数为 $1 \%$, 分离角 度的标准差均设为 $0.4^{\circ}$. 其他的模型参数如火箭入轨 参数、分离时间、卫星质量特性等均取标称值, 在此 基础上进行仿真分析.

表 1 四星分离时刻

\begin{tabular}{cc}
\hline 分离时序 & 时间 $(\mathrm{s})$ \\
\hline 点火发射 & $T_{0}$ \\
A星分离 & $T_{0}+813$ \\
B星分离 & $T_{0}+839$ \\
C星分离 & $T_{0}+868$ \\
D星分离 & $T_{0}+897$ \\
\hline
\end{tabular}


表 2 四星分离速度和分离角度

\begin{tabular}{ccccc}
\hline \multirow{2}{*}{ 卫星 } & 分离速度 $(\mathrm{m} / \mathrm{s})$ & \multicolumn{3}{c}{ 分离角度 $\left(^{\circ}\right)$} \\
\cline { 3 - 5 } & & 滚转 $(\mathrm{roll})$ & 俯仰 $(\mathrm{pitch})$ & 偏航(yaw) \\
\hline A星 & 0.66 & 2.562 & -35.23 & -0.180 \\
B星 & 0.448 & 2.554 & -43.636 & 0.267 \\
C 星 & 0.42 & 2.536 & -26.891 & -0.403 \\
D星 & 0.31 & 2.459 & -14.713 & 9.154 \\
\hline
\end{tabular}

分离安全性分析分为近场分析和远场分析. 近场 分析主要考虑卫星分离后在帆板展开前后是否有碰撞 危险; 远场分析主要考虑卫星在长期运行中是否发生 距离过于接近的情况. 将 $\mathrm{A}$ 星分离后 $200 \mathrm{~s}$ 以内定义为 近场分析时段，200 12000 s 定义为远场分析时段. 卫 星轨道外推模型采用 $\mathrm{J} 2$ 模型, 积分器为 ODE4, 积分步 长1 s. 在各星的分离速度和分离角度均取标称值的情 况下, A, B, C, D 4 颗星的星间距离在 1 天内的总体变化 趋势如图1(a)所示, 根据分析结果可知, 四星相对距离 总体上呈振荡增加趋势. 在此基础上, 关注分离后较短
时间内的星间距离, 得到近场分析和远场分析结果分 别如图1(b)和(c)所示. 由于星间距离存在振荡情况, 从 图1(c)可以看出, $\mathrm{B}$ 星和 $\mathrm{D}$ 星再接近之后的距离较小, 第 一次再接近发生在第3964 $\mathrm{s}$ (时间起点为 $\mathrm{A}$ 星分离时 刻), 两星距离最小达 $274.84 \mathrm{~m}$; 第二次再接近发生在 第8272 $\mathrm{s}$ (时间起点为 $\mathrm{A}$ 星分离时刻), 两星距离最小达 $135.69 \mathrm{~m}$, 此后B和D两星距离开始逐步振荡增加.

在四星的分离速度和分离角度均为随机变量的情 况下, 进一步分析四星分离后的星间距离. 由于该模型 共有 16 个随机变量, 变量维度较高, 传统的伪随机数生
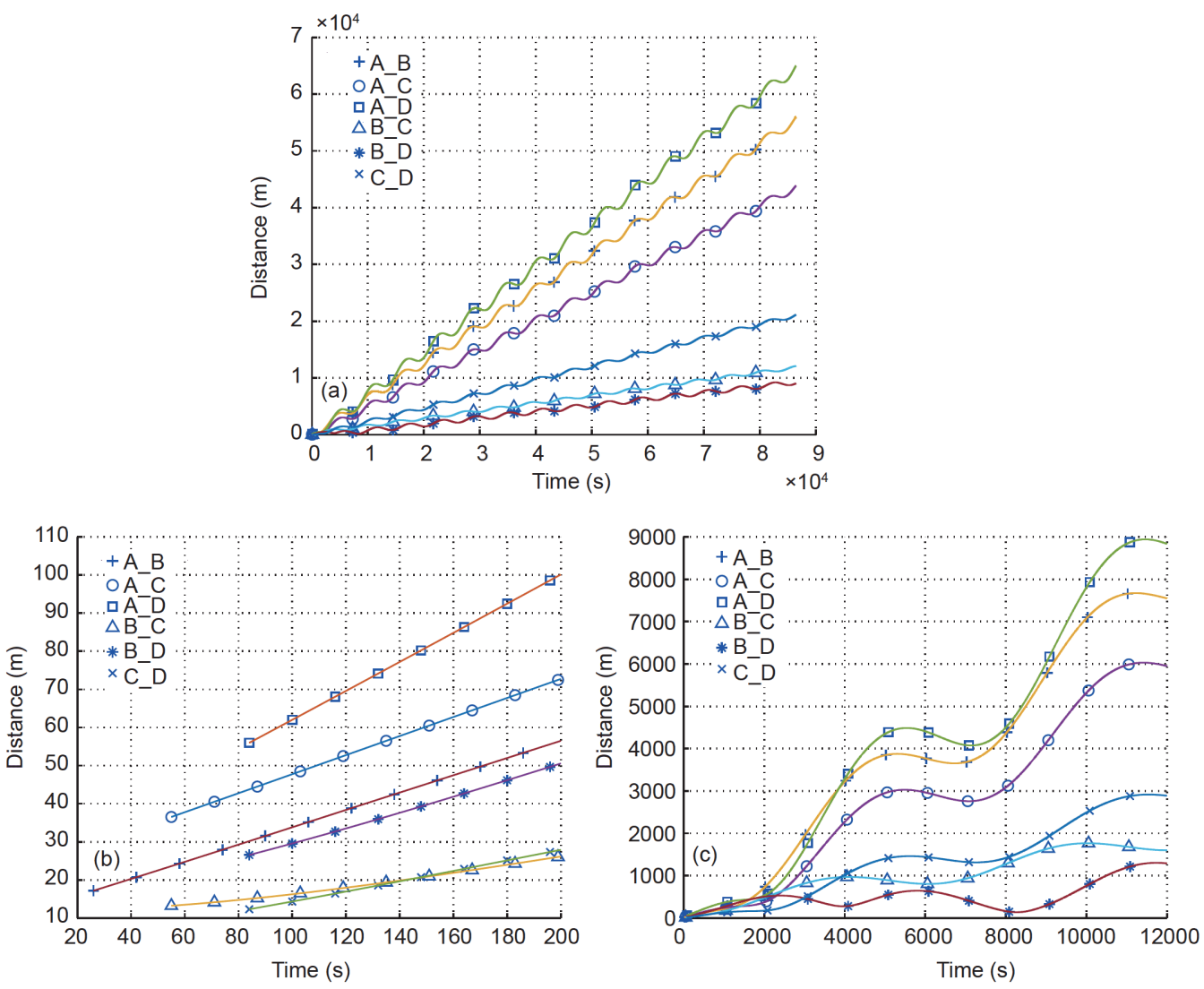

图 1 (网络版彩图)四星相对距离分析. (a) 1 天内相对距离分析; (b) 四星200 s以内距离分析; (c) 四星12000 s以内距离分析 
成器(pseudo-random number generator) ${ }^{[18]}$ 产生的实验 样本点在样本数较少或空间维度较高时会出现样本分 布与真实分布不一致的情况 ${ }^{[19]}$. 此种情况下，低偏差 抽样策略通常能得到具有较好收玫性质的样本，本文 利用目前最广泛使用的Latin超立方序列 ${ }^{[20]}$ 抽取实验 样本点, 样本规模为 10000 , 并计算每一个样本对应的 星间距离.

根据样本计算结果, 统计分析 $200 \mathrm{~s}$ 以内各颗星分 离后距离最小值 $\left(\mathrm{A}\right.$ 星与 $\mathrm{B}$ 星最小距离定义为 $d_{\mathrm{min}, \mathrm{A}-\mathrm{B}}$, 其他卫星间最小距离定义与此类似)、200 s之后各颗 星再次接近的距离极小值 $(\mathrm{A}$ 星与 $\mathrm{B}$ 星距离极小值定义 为 $d_{\text {min, L-A-B }}$, 其他卫星间距离极小值定义与此类似). 结 合图1(c)所示的分析结果，针对 $\mathrm{B}$ 星与 $\mathrm{D}$ 星需分析两次 再接近的距离极小值, 第一次接近的距离最小值定义 为 $d_{\min , \mathrm{L}-\mathrm{B}-\mathrm{D} 1}$, 第二次接近的距离最小值定义为

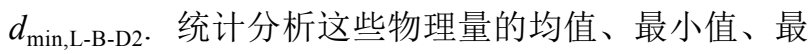
大值和标准差, 结果如表3所示.

根据表中结果分析，在200 s以内的近场分析阶段， 各颗星刚刚分离, 星间距离较近, 这是无法避免的, 但 从标准差结果和最小、最大值可知, 分离速度与分离 角度的偏差对星间距离的不确定性影响较小, 因此针 对这些距离量开展重要性测度分析意义不大．对于 200 12000 s 的远场分析阶段, 从均值情况看, B星和D 星的第一次接近和第二次接近的距离较小, 需要重点 关注. 此外, $\mathrm{C}$ 星和 $\mathrm{D}$ 星尽管距离均值较大，但最小值
仅 $143.32 \mathrm{~m}$, 说明该距离量的不确定性较强, 同样需要 重点关注.

\section{3 重要性测度指标定义及其高效求解}

\section{1 基于方差的重要性测度指标定义}

考虑如下的一般形式的模型方程:

$Y=g\left(X_{1}, X_{2}, \ldots, X_{n}\right)=g(\mathbf{X})$,

其中, $\mathbf{X}=\left(X_{1}, X_{1}, \ldots, X_{n}\right)$ 为相互独立的基本输入变量, $Y$ 为模型输出响应量. 同时假设模型功能函数 $g(\mathbf{X})$ 在输 入变量的定义域上平方可积. 则 $g(\mathbf{X})$ 的高维模型替代 展式可描述如下 ${ }^{[7]}$ :

$$
\begin{aligned}
& g\left(X_{1}, X_{2}, \ldots, X_{n}\right) \\
& \quad=g_{0}+\sum_{i} g_{i}+\sum_{i} \sum_{j>i} g_{i j}+\cdots+g_{12 \cdots n},
\end{aligned}
$$

式中, 每一项均为其下标所对应的输入变量的函数, 即 $g_{i}=g_{i}\left(X_{i}\right), g_{i j}=g_{i j}\left(X_{i}, X_{j}\right)$, 以此类推.

事实上, 高维模型替代展式并不唯一. 针对此问 题, Sobol ${ }^{[11]}$ 证明了如下结论: 如果高维模型替代展式 中每一项均值为零, 则该展式中各项之间两两正交且 各项能够被唯一确定. 各项表达式具体如下所示:

$$
\begin{aligned}
& g_{0}=E(Y), \\
& g_{i}=E\left(Y \mid X_{i}\right)-E(Y),
\end{aligned}
$$

\begin{tabular}{|c|c|c|c|c|c|}
\hline 阶段 & 物理量 & 均值 (m) & 最小值 (m) & 最大值 (m) & 标准差 (m) \\
\hline \multirow{6}{*}{$200 \mathrm{~s}$ 以内 } & $d_{\min , \mathrm{A}-\mathrm{B}}$ & 17.15 & 16.38 & 17.98 & 0.17 \\
\hline & $d_{\min , \mathrm{A}-\mathrm{C}}$ & 36.51 & 34.86 & 38.26 & 0.36 \\
\hline & $d_{\min , \mathrm{A}-\mathrm{D}}$ & 55.97 & 53.46 & 58.65 & 0.55 \\
\hline & $d_{\min , \mathrm{B}-\mathrm{C}}$ & 13.21 & 12.62 & 13.80 & 0.13 \\
\hline & $d_{\min , \mathrm{B}-\mathrm{D}}$ & 26.53 & 22.59 & 27.72 & 0.27 \\
\hline & $d_{\min , \mathrm{C}-\mathrm{D}}$ & 12.29 & 11.77 & 12.83 & 0.12 \\
\hline \multirow{7}{*}{$200 \sim 12000 \mathrm{~s}$} & $d_{\min , \mathrm{L}-\mathrm{A}-\mathrm{B}}$ & 3652.74 & 3114.44 & 4190.70 & 122.15 \\
\hline & $d_{\min , \mathrm{L}-\mathrm{A}-\mathrm{C}}$ & 2758.53 & 2190.86 & 3371.08 & 125.15 \\
\hline & $d_{\min , \mathrm{L}-\mathrm{A}-\mathrm{D}}$ & 4074.99 & 3529.34 & 4624.99 & 117.23 \\
\hline & $d_{\min , \mathrm{L}-\mathrm{B}-\mathrm{C}}$ & 803.82 & 372.05 & 1234.70 & 98.89 \\
\hline & $d_{\min , \mathrm{L}-\mathrm{B}-\mathrm{D} 1}$ & 144.97 & 22.59 & 313.96 & 80.36 \\
\hline & $d_{\min , \mathrm{L}-\mathrm{B}-\mathrm{D} 2}$ & 276.37 & 122.12 & 669.05 & 25.19 \\
\hline & $d_{\min , \mathrm{L}-\mathrm{C}-\mathrm{D}}$ & 1276.70 & 143.32 & 1702.65 & 234.46 \\
\hline
\end{tabular}

表 3 随机变量条件下星间距离统计结果 
$g_{i j}=E\left(Y \mid X_{i}, X_{j}\right)-g_{i}-g_{j}-E(Y)$,

参照功能函数的高维模型替代展式, 模型输出响 应量 $Y$ 的无条件方差 $V(Y)$ 同样可以进行类似的分解, 该 方差分解式可描述如下:

$V(Y)=\sum_{i} V_{i}+\sum_{i} \sum_{j>i} V_{i j}+\cdots+V_{12 \cdots n}$,

其中,

$$
\begin{aligned}
V_{i}= & V\left(E\left(Y \mid X_{i}\right)\right), \\
V_{i j}= & V\left(E\left(Y \mid X_{i}, X_{j}\right)\right)-V\left(E\left(Y \mid X_{i}\right)\right) \\
& -V\left(E\left(Y \mid X_{j}\right)\right),
\end{aligned}
$$$$
\cdots
$$

将式(6)的两边同除以模型输出响应量 $Y$ 的无条件 方差 $V(Y)$, 可得:

$1=\sum_{i} S_{i}+\sum_{i} \sum_{j>i} S_{i j}+\cdots+S_{12 \cdots n}$,

其中, $S_{i}=V_{i} / V(Y)$ 称为一阶灵敏度指标(也可称为输入变 量方差对输出响应量方差贡献的“主效应”), 该指标可 用于衡量输入变量 $X_{i}$ 对输出响应量方差的单独贡献. $S_{i j}=V_{i j} / V(Y)$ 可称为二阶灵敏度指标, 该指标可用于衡量 输入变量 $X_{i}$ 和 $X_{j}$ 之间的交互作用对输出响应量的方差 贡献; 其余各项的含义依此类推.

特别地, 我们介绍输入变量 $X_{i}$ 的总指标, 该指标解 释了变量 $X_{i}$ 对输出响应量方差的总贡献，包括一阶方 差贡献和高阶方差贡献. 该指标的定义如下所示:

$$
S T_{i}=S_{i}+\sum_{j>i} S_{i j}+\cdots+S_{12 \cdots n} .
$$

由式(9), 若直接计算某输入变量的总指标, 需要 计算各阶指标, 然后求和, 当模型输入变量个数很多 时，直接计算总指标则计算量过大，难以为工程实际 所接受. 实际上, 经过推导, 总指标的计算公式可简化 如下:

$$
S T_{i}=\frac{E\left(V\left(Y \mid \mathbf{X}^{(\sim i)}\right)\right)}{V(Y)}=1-\frac{V\left(E\left(Y \mid \mathbf{X}^{(\sim i)}\right)\right)}{V(Y)},
$$

其中, $\mathbf{X}^{(\sim i)}$ 为除 $X_{i}$ 之外的输入变量向量.

实际的重要性测度分析中，通常仅考虑一阶指标 $S_{i}$ 和总指标 $S T_{i}$.

\section{2 移动最小二乘方法}

移动最小二乘拟合的计算过程如下.

在某一局部拟合区域上，通过移动最小二乘法所 建立的拟合函数如下所示:

$$
\operatorname{fit}(\mathbf{X})=\sum_{i=1}^{m} \mathbf{a}_{i}(\mathbf{X}) p_{i}(\mathbf{X})=\mathbf{p}^{\mathrm{T}}(\mathbf{X}) \mathbf{a}(\mathbf{X}),
$$

其中, $\mathbf{a}(\mathbf{X})=\left[a_{1}(\mathbf{X}), a_{2}(\mathbf{X}), \ldots, a_{m}(\mathbf{X})\right]^{\mathrm{T}}$ 为待求系数向量, 该向量随着拟合区域的变化而变化, 因而它是变量 $\mathbf{X}$

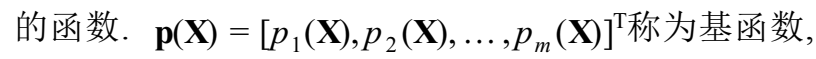
$m$ 是基函数的项数. 基函数通常按如下两种方式选取:

线性 : $\mathbf{p}(\mathbf{X})=\left[1, X_{1}, \ldots, X_{n}\right]^{\mathrm{T}}$,

二次 : $\mathbf{p}(\mathbf{X})=\left[1, X_{1}, \ldots, X_{n}, X_{1}^{2}, \ldots, X_{n}^{2}\right]^{\mathrm{T}}$,

其中, $n$ 为输入随机变量的个数.

移动最小二乘估计的误差可通过如下所示的加权 离散 $L_{2}$ 范式表示:

$$
\begin{aligned}
J & =\sum_{I=1}^{N_{\text {in }}} w\left(\mathbf{X}-\mathbf{X}_{I}\right)\left[\mathrm{fit}\left(\mathbf{X}_{I}, \mathbf{X}\right)-Y_{I}\right]^{2} \\
& =\sum_{I=1}^{N_{\text {in }}} w\left(\mathbf{X}-\mathbf{X}_{I}\right)\left[\mathbf{p}^{\mathrm{T}}\left(\mathbf{X}_{I}\right) \mathbf{a}(\mathbf{X})-Y_{I}\right]^{2},
\end{aligned}
$$

其中, $N$ 为当前迭代过程中影响区域内实验点的数目, $\operatorname{fit}\left(\mathbf{X}_{I}, \mathbf{X}\right)$ 为拟合函数, $Y_{I}$ 为实际函数(显式或隐式)在实 验点 $\mathbf{X}_{I}$ 处的真实值, $w\left(\mathbf{X}-\mathbf{X}_{I}\right)$ 为实验点 $\mathbf{X}_{I}$ 处的权函数 (可采用三次样条函数). 为了使移动最小二乘估计的 误差达到最小, 系数向量 $\mathbf{a}(\mathbf{X})$ 应满足如下关系式:

$\frac{\partial J}{\partial \mathbf{a}}=\mathbf{A}(\mathbf{X}) \mathbf{a}(\mathbf{X})-\mathbf{B}(\mathbf{X}) \mathbf{Y}=0$.

从上式我们可以求得如下的系数向量表达式:

$\mathbf{a}(\mathbf{X})=\mathbf{A}^{-1}(\mathbf{X}) \mathbf{B}(\mathbf{X}) \mathbf{Y}$,

其中,

$$
\begin{aligned}
& \mathbf{A}(\mathbf{X})=\sum_{I=1}^{N_{\mathrm{in}}} w\left(\mathbf{X}-\mathbf{X}_{I}\right) \mathbf{p}\left(\mathbf{X}_{I}\right) \mathbf{p}^{\mathrm{T}}\left(\mathbf{X}_{I}\right), \\
& \mathbf{B}(\mathbf{X})=\left[w\left(\mathbf{X}-\mathbf{X}_{1}\right) \mathbf{p}\left(\mathbf{X}_{1}\right), \ldots, w\left(\mathbf{X}-\mathbf{X}_{n}\right) \mathbf{p}\left(\mathbf{X}_{n}\right)\right], \\
& \mathbf{Y}^{\mathrm{T}}=\left[Y_{1}, Y_{2}, \ldots, Y_{n}\right] .
\end{aligned}
$$

将式(15)代入式(11), 我们可以求得移动最小二乘 估计的拟合函数如下: 


$$
\operatorname{fit}(\mathbf{X})=\sum_{I=1}^{N_{\text {in }}} \Phi_{I}^{k}(\mathbf{X}) Y_{I}=\ddot{\mathbf{O}}^{k}(\mathbf{X}) \mathbf{Y},
$$

其中, $k$ 表示基函数的阶数, $\ddot{\mathbf{O}}^{k}(\mathbf{X})$ 称为形函数:

$$
\begin{aligned}
\ddot{\mathbf{O}}^{k}(\mathbf{X}) & =\left[\Phi_{1}^{k}, \Phi_{2}^{k}, \cdots, \Phi_{n}^{k}\right] \\
& =\mathbf{p}^{\mathrm{T}}(\mathbf{X}) \mathbf{A}^{-1}(\mathbf{X}) \mathbf{B}(\mathbf{X}) .
\end{aligned}
$$

\section{3 指标高效求解新方法}

基于移动最小二乘拟合的重要性测度分析方法的 执行步骤如下.

(1) 根据基本输入变量的分布参数, 生成样本量为 $N$ 的一组随机样本, 并将这些样本组合成如下形式的 样本矩阵:

$\mathbf{S X}=\left(\mathbf{x}_{1}, \ldots, \mathbf{x}_{n}\right)=\left[\begin{array}{ccc}x_{11} & \ldots & x_{n 1} \\ \vdots & \ddots & \vdots \\ x_{1 N} & \cdots & x_{n N}\end{array}\right]$,

其中, 矩阵 $\mathbf{S X}$ 的第 $i$ 列表示输入变量 $X_{i}$ 的 $N$ 个随机样 本值.

(2) 计算步骤(1)中生成的 $N$ 组样本对应的模型输 出响应量值:

$$
\mathbf{Y}=\left(\begin{array}{c}
Y_{1} \\
\vdots \\
Y_{N}
\end{array}\right)=\left(\begin{array}{l}
g\left(x_{11}, \ldots, x_{i 1}, \ldots, x_{n 1}\right) \\
\vdots \\
g\left(x_{1 N}, \ldots, x_{i N}, \ldots, x_{n N}\right)
\end{array}\right) .
$$

模型输出响应量的总方差(即无条件方差) 估计 值 $\widehat{V Y}$ 可按下式计算:

$$
\widehat{V Y}=V(Y)=\frac{1}{N-1} \sum_{j=1}^{N}\left(Y_{j}-\operatorname{mean}(Y)\right)^{2},
$$

式中, 算子mean(•)表示求期望.

(3) 应用移动最小二乘拟合估计条件期望 $E\left(Y \mid X_{i}\right)$ 和输入变量 $X_{i}$ 之间的近似解析关系, 拟合函数如下 所示:

$$
E Y=E\left(Y \mid X_{i}\right)=\ddot{\mathbf{O}}^{k}\left(X_{i}\right) \mathbf{Y}_{\text {inner }}^{\left(X_{i}\right)}+e,
$$

式中, $\ddot{\mathbf{O}}^{k}\left(X_{i}\right)$ 为输入变量 $X_{i}$ 的形函数, $\mathbf{Y}_{\text {inner }}^{\left(X_{i}\right)}$ 为影响区域 内实验样本点的真实响应值向量, $e$ 为拟合误差.

文献[15]指出, 在最小二乘意义下, 原函数的最佳 单变量逼近函数 (以 $X_{i}$ 为自变量)即为条件期望 $E\left(Y \mid X_{i}\right)$.

(4) 条件期望 $E\left(Y \mid X_{i}\right)$ 的方差 $V\left(E\left(Y \mid X_{i}\right)\right)$ 可按下式
估计:

$$
\begin{aligned}
\widehat{V Y_{i}} & =V\left(E\left(Y \mid X_{i}\right)\right. \\
& =\frac{1}{N-1} \sum_{j=1}^{N}\left(\widehat{E Y}_{j}^{(i)}-\operatorname{mean}(E Y)\right)^{2},
\end{aligned}
$$

式中, $\overline{E Y}_{j}^{(i)}=\ddot{\mathbf{O}}^{k}\left(X_{j i}\right) \mathbf{Y}_{\text {inner }}^{\left(X_{j i}\right)}+e(i=1,2, \ldots n, j=1,2, \ldots, N)$.

(5) 根据式(23)和(25), 一阶重要性测度指标 $S_{i}$ $(i=1,2, \ldots n)$, 即变量 $X_{i}$ 的主贡献, 可按下式估计:

$\widehat{S}_{i}=\frac{\widehat{V Y}_{i}}{\widehat{V Y}}$

(6) 类似地, 我们可以采用移动最小二乘法估计输 出响应量关于多个输入变量的多重条件期望, 进而计 算高阶重要性测度指标. 但工程实际中, 工程师通常 更关注某输入变量对输出响应量方差的总贡献, 即需 要计算式(10)所定义的总指标. 首先仍采用移动最小 二乘法来拟合条件期望 $E\left(Y \mid \mathbf{X}^{(\sim i)}\right)$ 和除 $X_{i}$ 之外的所有输 入变量 $\left(\right.$ 用 $\mathbf{X}^{(\sim i)}$ 表示)之间的近似函数关系, 如下所示:

$$
E Y=E\left(Y \mid \mathbf{X}^{(\sim i)}\right)=\ddot{\mathbf{O}}^{k}\left(\mathbf{X}^{(\sim i)}\right) \mathbf{Y}_{\text {inner }}^{\left(\mathbf{X}^{(\sim i)}\right)}+e,
$$

式中, $\mathbf{X}^{(\sim i)}=\left(X_{i}, \ldots, X_{i-1}, X_{i+1}, \ldots, X_{n}\right)$ 为除 $X_{i}$ 之外的所有 输入变量组成的向量, $\ddot{\mathbf{O}}^{k}\left(\mathbf{X}^{(\sim i)}\right)$ 为参数 $\mathbf{X}^{(\sim i)}$ 的形函数,

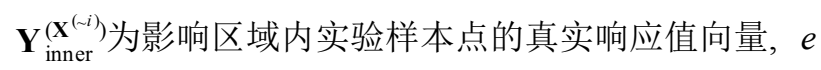
为拟合误差.

(7) 求解条件期望的方差 $V\left(E\left(Y \mid \mathbf{X}^{(\sim i)}\right)\right)$ 如下所示:

$$
\begin{aligned}
\widehat{V Y}^{(\sim i)} & =V\left(E\left(Y \mid \mathbf{X}^{(\sim i)}\right)\right. \\
& =\frac{1}{N-1} \sum_{j=1}^{N}\left(\widehat{E Y}_{j}^{(\sim i)}-\operatorname{mean}(E Y)\right)^{2},
\end{aligned}
$$

式中, $\overline{E Y_{j}^{(\sim i)}}=\ddot{\mathbf{O}}^{k}\left(\mathbf{X}_{j}^{(\sim i)}\right) \mathbf{Y}_{\text {inner }}^{\left(\mathbf{X}_{j}^{(-i)}\right)}+e$, 且 $\mathbf{X}_{j}^{(\sim i)}=\left(X_{j 1}, \ldots\right.$, $\left.X_{j, i-1}, X_{j, i+1}, \ldots, X_{j n}\right)(j=1,2, \ldots, N)$.

(8) 根据式(10), (23)和(28), 变量 $X_{i}$ 的总指标为

$$
\widehat{S T_{i}}=1-\frac{\widehat{V Y}^{(\sim i)}}{\widehat{V Y}} \text {. }
$$

\section{4 数值算例验证}

为验证基于移动最小二乘的重要性测度指标计算 的精度及效率, 考虑在重要性测度分析方法验证中经 常采用的Ishigami函数模型 ${ }^{[7]}$ 如下: 
$Y=\sin X_{1}+a \sin ^{2} X_{2}+b X_{3}^{4} \sin X_{1}$

式中, $X_{i} \sim U(-\pi, \pi), i=1,2,3$. 取 $a=7, b=0.1$.

Ishigami函数模型具有较高的非线性程度, 能够验 证求解方法对非线性模型的适应性. 此外, 采用Ishigami 模型的好处在于其存在解析解, 详细的求解过程可 查阅引用文献.

采用解析法、Saltelli方法 ${ }^{[14]}$ 和基于移动最小二乘 拟合的方法求解各变量的主指标和总指标, 所得结果 见表4.

由于该Ishigami函数模型功能函数较为复杂, 且非 线性程度较高, Saltelli方法在其样本量增大至 $10^{5}$ 时才 能得到较为稳定的结果，且不同变量的测度指标需要 重新抽取样本点, Saltelli方法总的计算代价为 $10^{5} \times(2$ $+2)=4 \times 10^{5}$. 而基于移动最小二乘拟合的方法只需采用 5000 个样本点便可较为精确地求得所有输入变量的一 阶主指标和总指标, 效率相比Saltelli方法有明显提高.

\section{4 分离安全性的重要性测度分析}

对于本文所描述的一箭四星分离模型, 根据第2节 分析结果, 需要开展重要性测度分析的物理量包括: (1) 远场分析阶段 $\mathrm{B}$ 星和 $\mathrm{D}$ 星第一次接近的最小距离 $d_{\text {min,L-B-D1 }}$ ；(2) 远场分析阶段 $\mathrm{B}$ 星和 $\mathrm{D}$ 星第二次接近的 最小距离 $d_{\text {min,L-B-D2 }}$ （3) 远场分析阶段 $\mathrm{C}$ 星和 $\mathrm{D}$ 星第一 次接近的最小距离 $d_{\text {min,L-C-D. }}$ 直接利用第2节中统计分 析所抽取的样本进行重要性测度计算, 无需额外抽取 样本, 得到的基本变量重要性测度结果如表5所示. 表 中第1列为模型所有的随机变量, $A_{\text {roll }}$ 为 $\mathrm{A}$ 星分离时刻 的滚转角、 $A_{\text {pitch }}$ 为 $\mathrm{A}$ 星分离时刻的俯仰角、 $A_{\text {yaw }}$ 为 $\mathrm{A}$ 星分离时刻的偏航角、 $A_{\mathrm{dv}}$ 为 $\mathrm{A}$ 星分离时刻的相对速 度, 其他各星的变量定义与此类似. 表中第2 列和第3列 为以 $d_{\text {min, L-B-D } 1}$ 为模型输出响应量的各基本变量的一阶 指标和总指标，第 4 列和第 5 列为以 $d_{\min , \mathrm{L}-\mathrm{B}-\mathrm{D} 2}$ 为模型输
出响应量的各基本变量的一阶指标和总指标，第6列 和第7列为以 $d_{\text {min,L-C-D }}$ 为模型输出响应量的各基本变量 的一阶指标和总指标.

为直观比较各基本变量的重要性测度指标大小, 针对不同的模型输出响应量绘制所有基本输入变量的 一阶指标和总指标柱形图如图2(a) (f)所示.

\section{5 分离安全性优化}

\section{1 星间距离分析及优化}

为直观表明模型输出响应量与重要性测度值较高 变量(定义为重要变量)之间的关系, 结合上一节重要 性测度分析结果, 绘制所关注的星间距离量与重要变 量之间的散点分布图, 并绘制重要性测度计算过程中 得到的对应变量的条件期望.

远场阶段 $\mathrm{B}$ 星和 $\mathrm{D}$ 星第一次接近的最小距离 $\left(d_{\min , \mathrm{L}-\mathrm{B}-\mathrm{D} 1}\right)$ 与各重要变量(包括 $\mathrm{B}$ 星分离时刻的俯仰角 $B_{\mathrm{pitch}} 、 \mathrm{~B}$ 星分离相对速度 $B_{\mathrm{dv}}$ 和 $\mathrm{D}$ 星分离相对速度 $D_{\mathrm{dv}}$ )

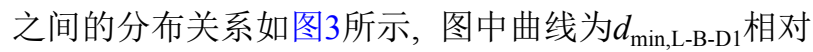
于各重要变量的条件期望值. 由图可见， B星和 $\mathrm{D}$ 星之 间的距离随着 $\mathrm{B}$ 星的俯仰角及分离速度的增大有增大 的趋势，而随 $\mathrm{D}$ 星分离速度的增大有减小趋势. 因此, 为保证星间距离，应尽量控制各个变量的分散性，同 时使 $\mathrm{B}$ 星的分离俯仰角及分离速度的分布集中于取值 较大的一侧, 并使 $\mathrm{D}$ 星分离速度的分布集中于取值较 小的一侧. 如此可以尽量保证 $\mathrm{B}$ 星和 $\mathrm{D}$ 星第一次接近的 最小距离不致过小，同时不会对其他的星间距离造成 不利影响. 实际工程中, 可通过笁选控制弹簧弹力、 爆炸螺栓冲击力, 优化星箭联合体姿控导引算法及分 离时间等对卫星的分离速度及分离角度进行有效 控制.

基于类似的分析过程，可以对远场阶段 $\mathrm{B}$ 星和 $\mathrm{D}$ 星 第二次接近的最小距离 $\left(d_{\text {min, L-B-D } 2}\right)$ 以及在远场阶段 $\mathrm{C}$ 星和 $\mathrm{D}$ 星第一次接近的最小距离 $\left(d_{\text {min,L-C-D }}\right)$ 与各个重要

表 4 Ishigami函数模型的重要性测度指标

\begin{tabular}{ccccccc}
\hline 方法 & $S_{1}$ & $S T_{1}$ & $S_{2}$ & $S T_{2}$ & $S_{3}$ & 0 \\
\hline 解析法 & 0.3139 & 0.5576 & 0.4424 & 0.4424 & 0.2437 & 0.2420 \\
Saltelli方法 & 0.3122 & 0.5456 & 0.4517 & 0.4373 & 0.0114 & 0.0032 \\
$\begin{array}{c}\text { 基于移动最小二乘的 } \\
\text { 方法 }\end{array}$ & 0.3140 & 0.5655 & 0.4389 & 0.4401 & 0.2507 \\
\hline
\end{tabular}


田龙飞等: 基于重要性测度的一箭多星分离安全性分析

表 5 一箭四星分离模型基本变量重要性测度

\begin{tabular}{ccccccc}
\hline \multirow{2}{*}{ 基本变量 } & \multicolumn{2}{c}{$d_{\text {min,L-B-D } 1}$} & \multicolumn{2}{c}{$d_{\text {min,L-B-D } 2}$} & \multicolumn{2}{c}{$d_{\text {min,L-C-D }}$} \\
\cline { 2 - 7 } & $S$ & $S T$ & $S$ & $S T$ & $S T$ \\
\hline$A_{\text {roll }}$ & $3.885 \times 10^{-4}$ & $2.042 \times 10^{-2}$ & $3.758 \times 10^{-4}$ & $1.497 \times 10^{-2}$ & $3.333 \times 10^{-4}$ & $6.340 \times 10^{-2}$ \\
$A_{\text {pitch }}$ & $4.144 \times 10^{-4}$ & $2.031 \times 10^{-2}$ & $1.517 \times 10^{-4}$ & $1.494 \times 10^{-2}$ & $3.451 \times 10^{-4}$ & $6.506 \times 10^{-2}$ \\
$A_{\text {yaw }}$ & $2.835 \times 10^{-4}$ & $1.991 \times 10^{-2}$ & $1.971 \times 10^{-4}$ & $1.474 \times 10^{-2}$ & $5.649 \times 10^{-4}$ & $6.196 \times 10^{-2}$ \\
$B_{\text {roll }}$ & $4.956 \times 10^{-4}$ & $2.013 \times 10^{-2}$ & $3.228 \times 10^{-4}$ & $1.540 \times 10^{-2}$ & $2.298 \times 10^{-4}$ & $6.390 \times 10^{-2}$ \\
$B_{\text {pitch }}$ & $2.400 \times 10^{-1}$ & $6.041 \times 10^{-2}$ & $8.699 \times 10^{-2}$ & $7.562 \times 10^{-2}$ & $2.407 \times 10^{-4}$ & $6.169 \times 10^{-2}$ \\
$B_{\text {yaw }}$ & $8.057 \times 10^{-4}$ & $2.037 \times 10^{-2}$ & $3.478 \times 10^{-4}$ & $1.427 \times 10^{-2}$ & $3.754 \times 10^{-4}$ & $6.380 \times 10^{-2}$ \\
$C_{\text {roll }}$ & $6.852 \times 10^{-4}$ & $2.001 \times 10^{-2}$ & $4.170 \times 10^{-4}$ & $1.465 \times 10^{-2}$ & $4.536 \times 10^{-4}$ & $6.282 \times 10^{-2}$ \\
$C_{\text {pitch }}$ & $4.220 \times 10^{-4}$ & $2.023 \times 10^{-2}$ & $3.813 \times 10^{-4}$ & $1.548 \times 10^{-2}$ & $1.489 \times 10^{-1}$ & $1.486 \times 10^{-1}$ \\
$C_{\text {yaw }}$ & $1.781 \times 10^{-3}$ & $2.049 \times 10^{-2}$ & $3.658 \times 10^{-4}$ & $1.480 \times 10^{-2}$ & $5.463 \times 10^{-4}$ & $6.416 \times 10^{-2}$ \\
$D_{\text {roll }}$ & $8.080 \times 10^{-4}$ & $2.043 \times 10^{-2}$ & $1.798 \times 10^{-4}$ & $1.487 \times 10^{-2}$ & $2.178 \times 10^{-4}$ & $6.359 \times 10^{-2}$ \\
$D_{\text {pitch }}$ & $2.079 \times 10^{-2}$ & $2.397 \times 10^{-2}$ & $2.019 \times 10^{-3}$ & $2.042 \times 10^{-2}$ & $3.622 \times 10^{-2}$ & $9.800 \times 10^{-2}$ \\
$D_{\text {yaw }}$ & $3.579 \times 10^{-3}$ & $2.074 \times 10^{-2}$ & $3.194 \times 10^{-3}$ & $1.793 \times 10^{-2}$ & $5.604 \times 10^{-4}$ & $6.269 \times 10^{-2}$ \\
$A_{\text {dv }}$ & $5.748 \times 10^{-4}$ & $2.032 \times 10^{-2}$ & $2.427 \times 10^{-4}$ & $1.537 \times 10^{-2}$ & $4.264 \times 10^{-4}$ & $6.502 \times 10^{-2}$ \\
$B_{\text {dv }}$ & $3.092 \times 10^{-1}$ & $6.813 \times 10^{-2}$ & $3.404 \times 10^{-1}$ & $1.584 \times 10^{-1}$ & $4.402 \times 10^{-4}$ & $6.202 \times 10^{-2}$ \\
$C_{\text {dv }}$ & $5.134 \times 10^{-4}$ & $2.017 \times 10^{-2}$ & $2.241 \times 10^{-4}$ & $1.480 \times 10^{-2}$ & $2.024 \times 10^{-1}$ & $1.545 \times 10^{-1}$ \\
$D_{\text {dv }}$ & $3.046 \times 10^{-1}$ & $6.888 \times 10^{-2}$ & $2.269 \times 10^{-1}$ & $1.235 \times 10^{-1}$ & $1.507 \times 10^{-1}$ & $1.447 \times 10^{-1}$ \\
\hline
\end{tabular}

变量的分布关系进行分析. 最终得到星间距离与重要 变量的相关性统计如表6所示，其中正相关表示星间 距离随变量的增大而增大，负相关表示星间距离随变 量的增大而减小.

基于上述分析结果，对重要变量的分布参数进行 调整, 调整结果如表7所示.

\section{2 优化结果}

根据新的基本变量分布参数, 重新利用Latin超立 方抽样策略生成新的样本, 样本规模仍为 10000 , 并计 算每一个样本组合对应的各星之间在近场阶段的最小 值和远场阶段的极小值，统计分析其均值、最小值、 最大值和标准差, 结果如表8所示.

由表 8 可见, 在对输入变量进行有针对性的调整之 后, 对于所关注的几颗星之间的距离得到显著增加, 且 不确定性得到控制, 不致出现星间距离过小的情况, 一 箭多星分离安全性得以保证.

\section{6 讨论与结论}

对于一箭多星发射任务, 入轨后的分离安全性对
于发射任务的成败至关重要，而分离后的各星之间的 距离是衡量分离安全性的重要指标. 入轨分离后各颗 卫星之间的相对距离受很多因素影响，需要对这些因 素的影响大小进行排序, 识别出重要影响因素, 从而 在实际工程研制过程中加以控制.

对于本文所涉及的一箭四星释放分离问题，其轨 道模型较为简单(圆轨、近距、短时间), 实际可以采 用形式上更为简便的 $\mathrm{CW}$ 方程开展分析，但 $\mathrm{CW}$ 方程在 模型复杂度增加后使用会受限, 因此, 本文采用了 J2摄 动模型进行轨道仿真分析. 对于本文的灵敏度分析过 程, 轨道相对动力学模型是可以自由选择的, 具有较 强的适应性.

重要性测度分析是当前国际可靠性及安全性研究 领域的热点方向, 众多学者分别提出了各自的指标体 系或高效计算方法. 本文利用基于方差的重要性测度 指标，提出了基于移动最小二乘的指标求解新方法, 相比传统的Monte Carlo法和Saltelli法等重要性测试指 标求解方法, 其计算效率在模型复杂度较高的情况下 优势十分明显，文中采用数值算例对新方法的计算精 度及效率进行了验证，结果表明所提方法具有较高的 求解精度及效率. 在此基础上, 将重要性测度分析方 

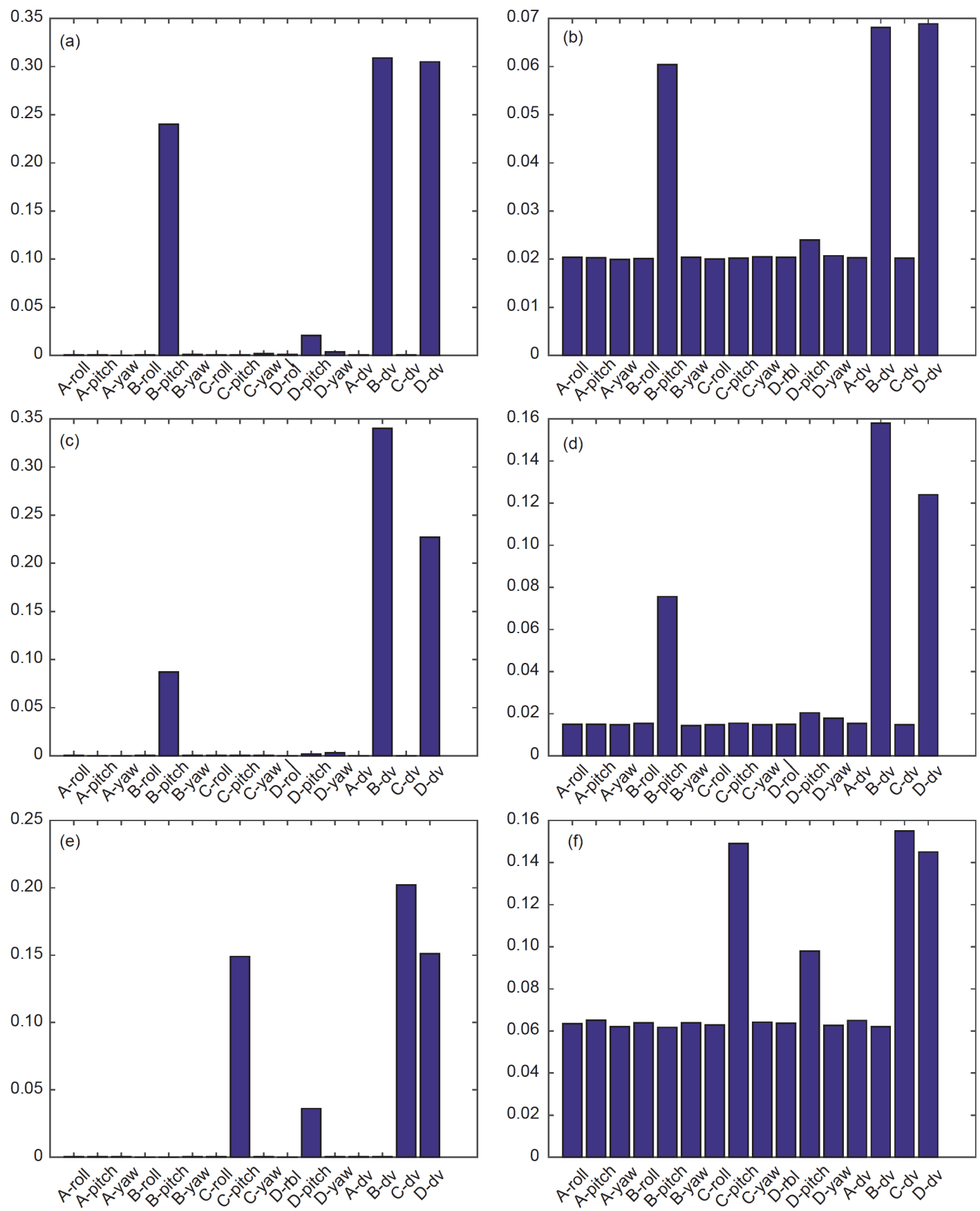

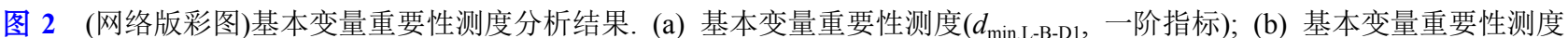
$\left(d_{\min , \mathrm{L}-\mathrm{B}-\mathrm{D} 1}\right.$, 总指标); (c) 基本变量重要性测度 $\left(d_{\min , \mathrm{L}-\mathrm{B}-\mathrm{D} 2}\right.$, 一阶指标); (d) 基本变量重要性测度 $\left(d_{\min , \mathrm{L}-\mathrm{B}-\mathrm{D} 2}\right.$, 总指标); (e) 基本变量重 要性测度 $\left(d_{\min , \mathrm{L}-\mathrm{C}-\mathrm{D}}\right.$, 一阶指标); (f) 基本变量重要性测度 $\left(d_{\text {min,L-C-D }}\right.$, 总指标)

法应用于一箭四星分离安全性分析，针对星间距离过 于接近的情况，计算基本输入变量的重要性测度，识 别出对星间距离影响较大的变量, 在此基础上, 对重 要变量的分布参数进行优化调整, 分析结果表明, 参
数优化后，所关注的星间距离明显增大，且不确定性 得到有效控制，从而保证了一箭四星的分离安全性. 对于实际的一箭多星航天发射任务，均可采用本文分 析方法开展相应研究, 提升任务可靠性及安全性. 

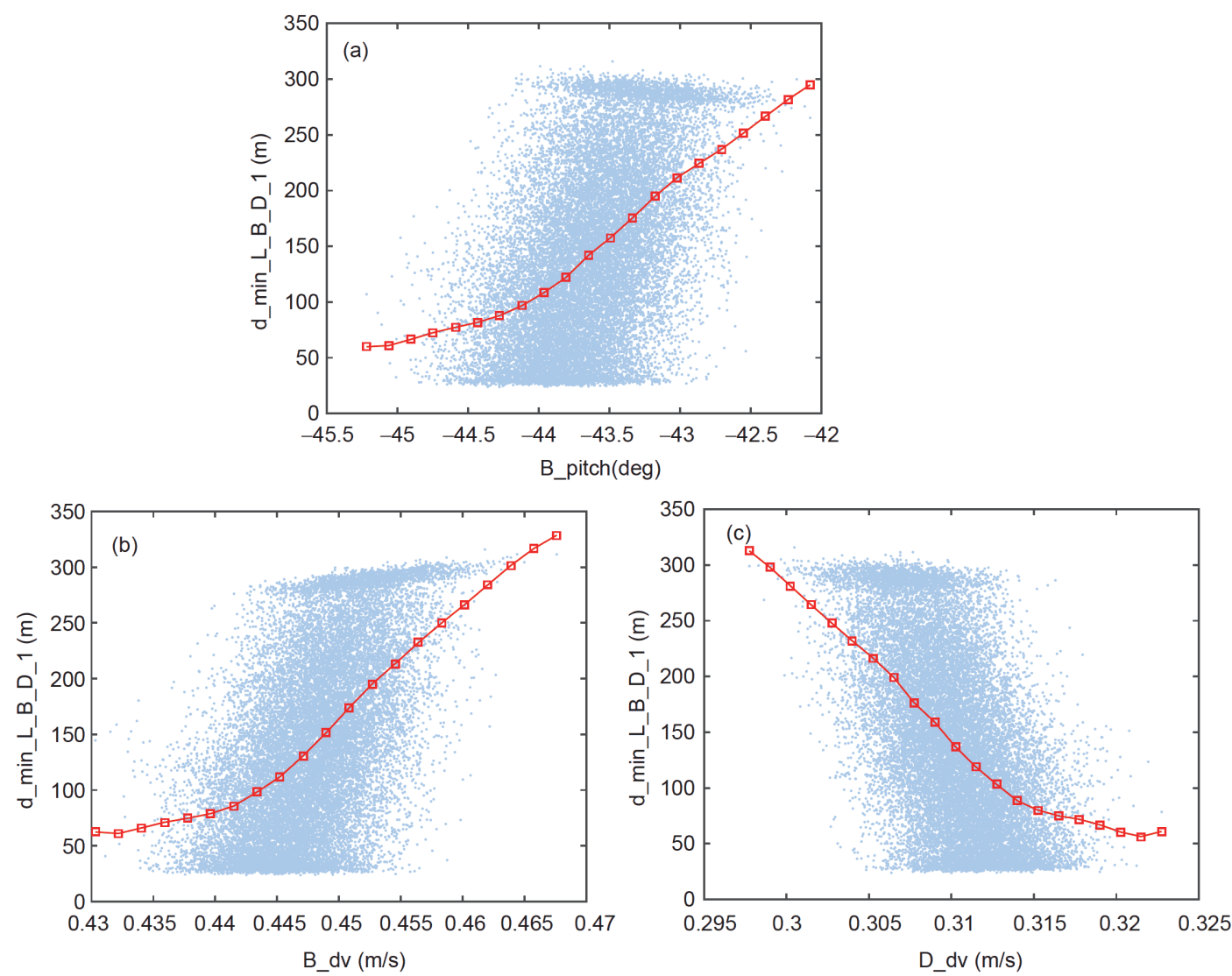

图 3 (网络版彩图)响应量 $d_{\text {min,L-B-D1 }}$ 与各重要变量分布散点图及条件期望. (a) 响应量与B星俯仰角关系; (b) 响应量与 $\mathrm{B}$ 星分离 速度关系; (c) 响应量与D星分离速度关系

表 6 星间距离量与各重要变量的相关性统计

\begin{tabular}{cccccc}
\hline 星间距离量 & $B_{\text {pitch }}$ & $C_{\text {pitch }}$ & $B_{\mathrm{dv}}$ & $C_{\mathrm{dv}}$ & $D_{\mathrm{dv}}$ \\
\hline$d_{\text {min,L-B-D1 }}$ & 正相关 & - & 正相关 & - & 负相关 \\
$d_{\text {min,L-B-D2 }}$ & 正相关 & - & 正相关 & - & 负相关 \\
$d_{\text {min,L-C-D }}$ & - & 正相关 & - & 正相关 & 负相关 \\
\hline
\end{tabular}

表 7 重要变量分布参数调整

\begin{tabular}{|c|c|c|c|c|}
\hline \multirow{2}{*}{ 重要变量 } & \multicolumn{2}{|c|}{ 调整前参数 } & \multicolumn{2}{|c|}{ 调整后参数 } \\
\hline & 均值 & 标准差 & 均值 & 标准差 \\
\hline$B_{\text {pitch }}\left({ }^{\circ}\right)$ & -43.636 & 0.4 & -42.5 & 0.15 \\
\hline$C_{\text {pitch }}\left({ }^{\circ}\right)$ & -26.891 & 0.4 & -25.5 & 0.15 \\
\hline$B_{\mathrm{dv}}(\mathrm{m} / \mathrm{s})$ & 0.448 & $4.48 \times 10^{-3}$ & 0.46 & $1.84 \times 10^{-}$ \\
\hline$C_{\mathrm{dv}}(\mathrm{m} / \mathrm{s})$ & 0.42 & $4.2 \times 10^{-3}$ & 0.43 & $1.72 \times 10^{-}$ \\
\hline$D_{\mathrm{dv}}(\mathrm{m} / \mathrm{s})$ & 0.31 & $3.1 \times 10^{-3}$ & 0.25 & $1.0 \times 10^{-3}$ \\
\hline
\end{tabular}


表 8 新随机变量条件下星间距离统计结果

\begin{tabular}{|c|c|c|c|c|c|}
\hline 阶段 & 物理量 & 均值 $(\mathrm{m})$ & 最小值 (m) & 最大值 (m) & 标准差 $(\mathrm{m})$ \\
\hline \multirow{6}{*}{$200 \mathrm{~s}$ 以内 } & $d_{\min , \mathrm{A}-\mathrm{B}}$ & 17.16 & 16.56 & 17.76 & 0.17 \\
\hline & $d_{\min , \mathrm{A}-\mathrm{C}}$ & 36.52 & 35.26 & 37.78 & 0.36 \\
\hline & $d_{\min , \mathrm{A}-\mathrm{D}}$ & 55.99 & 54.07 & 57.92 & 0.55 \\
\hline & $d_{\min , \mathrm{B}-\mathrm{C}}$ & 13.56 & 13.35 & 13.76 & 0.05 \\
\hline & $d_{\min , \mathrm{B}-\mathrm{D}}$ & 27.25 & 26.83 & 27.64 & 0.11 \\
\hline & $d_{\min , \mathrm{C}-\mathrm{D}}$ & 12.59 & 12.41 & 12.76 & 0.05 \\
\hline \multirow{7}{*}{$200 \sim 12000 \mathrm{~s}$} & $d_{\min , \mathrm{L}-\mathrm{A}-\mathrm{B}}$ & 3396.35 & 3002.37 & 3471.58 & 105.89 \\
\hline & $d_{\text {min,L-A-C }}$ & 2518.72 & 2155.52 & 2839.67 & 108.01 \\
\hline & $d_{\min , \mathrm{L}-\mathrm{A}-\mathrm{D}}$ & 5069.33 & 4740.61 & 5399.67 & 105.84 \\
\hline & $d_{\min , \mathrm{L}-\mathrm{B}-\mathrm{C}}$ & 775.71 & 648.38 & 903.88 & 39.35 \\
\hline & $d_{\min , \mathrm{L}-\mathrm{B}-\mathrm{D} 1}$ & 349.01 & 337.87 & 360.05 & 3.18 \\
\hline & $d_{\min , \mathrm{L}-\mathrm{B}-\mathrm{D} 2}$ & 1556.93 & 1438.48 & 1671.48 & 36.63 \\
\hline & $d_{\min , \mathrm{L}-\mathrm{C}-\mathrm{D}}$ & 2548.91 & 2443.48 & 2655.70 & 33.72 \\
\hline
\end{tabular}

本文假设各卫星分离速度与分离角度为相互独立 变量, 并在此基础上开展了重要性测度分析. 对于工程 实际, 随着统计数据研究的逐步深入, 变量之间可能呈
现一定的相关特性, 此时, 本文提出的重要性测度分析 方法不再适用, 需要进一步研究针对非独立变量的重 要性测度分析手段.

\section{参考文献}

1 谢长雄, 徐月同. 皮卫星星箭分离机构运动系统设计. 宇航学报, 2014, 35: 626-632

2 滕来, 金仲和. 皮卫星分离参数优化. 宇航学报, 2016, 37: 1200-1206

3 Santoni F, Piergentili F, Ravaglia R. Nanosatellite cluster launch collision analysis. J Aerosp Eng, 2013, 26: 618-627

4 Wen C, Zhang H, Gurfil P. Orbit injection considerations for cluster flight of nanosatellites. J Spacecr Rockets, 2015, 52: 196-208

5 Jiang C, Wang Z, Zhang Y. Development of the new approach of formation initialization using spring separation mechanism considering J2 perturbation. Adv Space Res, 2015, 55: 2616-2627

6 王金昌, 问波, 张佳, 等. 基于虚拟样机的多星分离仿真分析. 中国空间科学技术, 2016, 36: 70-76

7 Saltelli A, Ratto M, Andres T, et al. Global Sensitivity Analysis the Primer. West Sussex: John Wiley \& Sons Ltd, 2008. 155-169

8 McRae G J, Tilden J W, Seinfeld J H. Global sensitivity analysis-A computational implementation of the Fourier Amplitude Sensitivity Test (FAST). Comput Chem Eng, 1982, 6: 15-25

9 Li G, Rabitz H, Yelvington P E, et al. Global sensitivity analysis for systems with independent and/or correlated inputs. J Phys Chem A, 2010, 114: 6022-6032

10 Saltelli A, Scott M. Guest editorial: The role of sensitivity analysis in the corroboration of models and its link to model structural and parametric uncertainty. Reliab Eng Syst Saf, 1997, 57: 1-4

11 Sobol' I M. Global sensitivity indices for nonlinear mathematical models and their Monte Carlo estimates. Math Comput Simul, 2001, 55: 271280

12 Helton J C, Johnson J D, Sallaberry C J, et al. Survey of sampling-based methods for uncertainty and sensitivity analysis. Reliab Eng Syst Saf, 2006, 91: 1175-1209

13 Borgonovo E. A new uncertainty importance measure. Reliab Eng Syst Saf, 2007, 92: 771-784

14 Saltelli A. Making best use of model evaluations to compute sensitivity indices. Comput Phys Commun, 2002, 145: 280-297

15 Oakley J E, O’Hagan A. Probabilistic sensitivity analysis of complex models: A Bayesian approach. J R Statist Soc B, 2004, 66: 751-769 

6109

\title{
Study on safety of multi-satellite launch mission based on importance measure
}

\author{
TIAN LongFei ${ }^{1,2}$, LI Hua ${ }^{1}$, LIU Wu ${ }^{1,2}$, LIU Yong ${ }^{1}$, HU HaiYing ${ }^{1} \&$ LI PingFu $^{1}$ \\ ${ }^{1}$ Innovation Research Institude, Chinese Academy of Sciences, Shanghai 201203, China; \\ ${ }^{2}$ Shanghai Engineering Center of Micro-Satellite, Shanghai 201203, China
}

For multi-satellite launch mission, the velocity and direction of each satellite at separation may deviate from nominal value, which will introduce uncertainty to the distances between satellites after separation, and influence the safety as a result. In order to improve the satellite rocket separation safety of multi-satellite launch mission, importance measure analysis technology is employed to analyze the distance between satellites after separation. A new method is proposed to calculate the importance measures, numerical example shows that the efficiency of the proposed method is higher than the traditional Monte Carlo method and Saltelli's method. For the situation that distance between satellites is too close after separation, importance measure analysis technology can be used to identify the input variables those have greater influence on the distances between satellites, then the distribution parameters of those input variables can be optimized, which can increase the minimum distance between satellites, and decrease its uncertainty as well. As a result, the safety during separation can be insured.

multi-satellite launch mission, separation safety, importance measure, moving-least squares approximation, uncertainty analysis

doi: $10.1360 /$ N092018-00145 